\title{
Dental MRI: a road beyond CBCT
}

\author{
Takashi Nakamura ${ }^{1}$
}

Received: 16 August 2020 / Revised: 16 August 2020 / Accepted: 18 September 2020 / Published online: 30 September 2020

(C) European Society of Radiology 2020

\section{Key Points}

- Three-dimensional (3D) imaging coupled with computer-guided surgery planning is the core of the contemporary dental implant practice.

- Magnetic resonance (MR)-based dental implant planning can achieve results comparable to those with cone beam computed tomography (CBCT)-based planning.

- MR-based dental implant planning without radiation dose could be a potential alternative to CBCT-based planning.

Abbreviations

MSVAT-SPACE Multiple-slab acquisition with view angle tilting gradient, based on a sampling perfection with application-optimised contrasts using different flip angle evolution

Dental implants restore the normal or near-normal occlusal function in partially or totally edentulous patients. Despite the fact that a primary barrier to receive dental implant is the high cost, a need for dental implant has been increasing among patients with missing tooth/teeth, along with recent advances in implant technology that are critical to safe procedures and satisfactory outcomes [1]. A dental implant is typically a titanium screw or cylinder, the surface of which is expected to be integrated by living bone through osteoblastic reactions in the surrounding host tissues (referred to as osseointegration) [2]. The surface may be coated with bioactive materials such as hydroxyapatite and calcium phosphate, or micro/nano-structured, both facilitating biological and biophysical interactions with the host tissues. An intraosseous implant supports a dental prosthesis, such as a ceramic crown, which is screw-fixed or cement-bonded to the implant through an abutment. Thus, successful dental implant surgery depends

This comment refers to the article available at https://oi.org/10.1007/ s00330-020-07262-1.

Takashi Nakamura

taku@nagasaki-u.ac.jp

1 Nagasaki University Graduate School of Biomedical Sciences, 1-7-1 Sakamoto, Nagasaki 852-8588, Japan heavily on the amounts of intact bone tissues adjacent to the intraosseous implant.

To draw up a meticulous plan for dental implant, three-dimensional (3D) imaging of the edentulous jaw bone area is indispensable. To achieve this, we have two options at present: multi-slice computed tomography (CT) or cone beam computed tomography (CBCT). Although CBCT lacks sufficient soft tissue contrast and standardized gray scale value, oral surgeons prefer to use CBCT rather than conventional multi-slice $\mathrm{CT}$. This is because CBCT is more accessible than conventional $\mathrm{CT}$ in that $\mathrm{CBCT}$ is smaller-sized, of lower costs, and easier to operate, compared with conventional CT. In addition, CBCT provides $3 \mathrm{D}$ images with voxel sizes comparable with or smaller than those for conventional $\mathrm{CT}$, thereby overriding the needs of conventional CT [3]. Accordingly, CBCT coupled with computerguided planning permits implant designs appropriate for a particular implant site, in terms of implant type (shape and surface structure), dimensions (length and diameter), and placement (entry point and angle) and also allows surgeons to decide the need for and type of bone augmentation (such as bone chip/split and sinus lift) for a particular edentulous region lacking sufficient amount of bone to support the implant. However, radiation dose with CBCT, especially for the thyroid, remains highly problematic, even though lower than that of conventional CT $[4,5]$.

In this issue, Hilgenfeld et al illustrated the usefulness of magnetic resonance (MR) imaging in dental implant planning [6]. In this prospective study, the authors evaluated the clinical feasibility of 3-T MR imaging for dental implant planning by comparing results between MR- and CBCT-based computerguided planning at 45 implant sites from 30 patients. The 
authors showed that MR-based planning did not require any further adjustments based on CBCT-based planning for implant type and dimensions in 43 of the 45 sites. A few minor adjustments, however, were needed for the implant position of 13 sites and the implant axis of 3 sites. Notably, 3D deviations in MR-based planning from CBCT-based planning fell within the range of $0.2-3.0 \mathrm{~mm}$ (mean $=1.1 \pm 0.7 \mathrm{~mm}$ ) at the entry point and of $0.2-3.0 \mathrm{~mm}(1.3 \pm 0.7 \mathrm{~mm})$ at the apex of implant, and angle deviations fell into a range between 0 and $6.6^{\circ}$ $\left(2.4 \pm 1.5^{\circ}\right)$. Furthermore, MR-based planning correctly judged whether or not bone augmentation is necessary in 42 of the 45 sites, with a positive predictive value of $100 \%$. Collectively, these data suggest that MR-based planning is sufficiently accurate for practical purposes.

Several attempts have been made to evaluate the reliability of MR imaging in dental implant planning [7, 8]. Unlike the current study, however, most of the preceding studies have not assessed the reliability and accuracy of MR-based planning in clinical workflow settings. Furthermore, the authors have exploited recently developed MR imaging techniques, including a toothsurface reconstruction algorithm, allowing for the integration of MR imaging data directly into a digital workflow without any need for additional software or time-consuming post-processing steps, and a smallsized surface coil specialized for dental use, instead of a standard head and neck coil, providing isotropic images with a small voxel size $(440 \mu \mathrm{m})$. Together with an MR sequence optimized to obtain high spatial resolution and to effectively suppress susceptibility artifacts from dental metals (that is, MSVAT-SPACE), these advanced techniques may have substantially improved the image quality and consequently the accuracy of dental implant planning.

Hilgenfeld et al raised several limitations of the proposed MR-based implant planning system and suggested a need for improving the accuracy before further clinical trials [6]. Some clinicians and researchers may be more downbeat to think that the clinical application of MRbased dental implant planning is far-fetched. It may indeed be hard to imagine that MR imaging with lower accessibility and spatial resolution compared with CBCT could supersede the latter in dental implant planning. However, radiation dose with CBCT, especially for younger generations, is a serious concern [5]. Furthermore, besides improving the accuracy, reliability, and usability of MR-based dental implant planning, MR-borne advantages over CBCT could also facilitate the clinical application of MR-based planning. Consistent with this notion, previous studies have shown that MR imaging can reliably assess bone water concentrations, bone marrow compositions, and bone mineral densities, all of which could influence the quality of bone tissues that are required for adequate host reactions and rigid supports for dental implants $[9,10]$.

CBCT is in the middle of the game and will continue as the main imaging modality in dental implant planning for the time being. The current work by Hilgenfeld and colleagues has shed light on the potential use of high-resolution MR imaging in the planning, clearly showing that MR-based planning can achieve results comparable to those with CBCT-based planning. However, some additional hardware and/or software innovations would be necessary for the clinical use of MRbased planning, thereby allowing stringent backward planning and improving the safety and efficiency of surgical procedures. This thorny issue must be tackled in a sophisticated way before radiologists and surgeons can embark on exploring how the new setting of MR imaging might work in the dental implant clinics.

Funding The authors state that this work has not received any funding.

\section{Compliance with ethical standards}

Guarantor The scientific guarantor of this publication is Takashi Nakamura.

Conflict of interest The author of this manuscript declares no relationships with any companies whose products or services may be related to the subject matter of the article.

Statistics and biometry No complex statistical methods were necessary for this paper.

Informed consent Written informed consent was waived by the Institutional Review Board.

Ethical approval Institutional Review Board approval was not required because this article is an editorial comment.

Methodology • Editorial comment

\section{References}

1. Elani HW, Starr JR, Da Silva JD, Gallucci GO (2018) Trends in dental implant use in the U.S., 1999-2016, and projections to 2026. J Dent Res 97:1424-1430

2. Meng HW, Chien EY, Chien HH (2016) Dental implant bioactive surface modifications and their effects on osseointegration: a review. Biomarker Res 4:24

3. Jacobs R, Salmon B, Codari M, Hassan B, Bornstein MM (2018) Cone beam computed tomography in implant dentistry: recommendations for clinical use. BMC Oral Health 18:88

4. Tsapaki V (2017) Radiation protection in dental radiology — recent advances and future directions. Phys Med 44:222-226

5. Stratis A, Zhang G, Jacobs R, Bogaerts R, Bosmans H (2019) Growing concern of radiation dose in paediatric dental and maxillofacial CBCT: an easy guide for dental practice. Eur Radiol 29: 7009-7018 
6. Hilgenfeld T, Juerchott A, Jende JME et al (2020) Use of dental MRI for radiation-free guided dental-implant planning: a prospective, in-vivo study of accuracy and reliability. Eur Radiol. https:// doi.org/10.1007/s00330-020-07262-1

7. Mercado F, Mukaddam K, Filippi A, Bieri OP, Lambrecht TJ, Kühl S (2019) Fully digitally guided implant surgery based on magnetic resonance imaging. Int J Oral Maxillofac Implants 34:529-534

8. Flügge T, Ludwig U, Hövener JB, Kohal R, Wismeijer D, Nelson K (2020) Virtual implant planning and fully guided implant surgery using magnetic resonance imaging — proof of principle. Clin Oral Implants Res 31:575-583
9. Techawiboonwang A, Song HK, Leonard MB, Wehrli FW (2008) Cortical bone water: in vivo quantification with ultrashort echotime MR imaging. Radiology 248:824-833

10. Chang G, Boone S, Martel D, et al (2017) MRI assessment of bone structure and microarchitecture. J Magn Reson Imaging 46:323337

Publisher's note Springer Nature remains neutral with regard to jurisdictional claims in published maps and institutional affiliations. 